\title{
A New Non-radial Network DEA Model for Evaluating Performance Supply Chain
}

\author{
Mohsen Rostamy-Malkhalifeh', Elahe Mollaeian', Somayeh Mamizadeh-Chatghayeh ${ }^{2 *}$ \\ ${ }^{1}$ Department of Mathematics, Science and Research Branch, Islamic Azad University, \\ Tehran, Iran; Mohsen_Rostamy@yahoo.com, elahemollaeian@yahoo.com \\ ${ }^{2}$ Young Researches Club, Islamic Azad University, Central Tehran branch, \\ Tehran,Iran; somayeh_mamizadeh@yahoo.com
}

\begin{abstract}
Data Envelopment Analysis (DEA) is a mathematic technique for measuring the efficiency of Decision Making Units (DMUs) with multiple inputs and outputs. The evaluation of performance is the most important duty in supply chain management and inform of relevant units' performance. In regard to complex structure which exist in some supply chains, must define the scale of performance for it so as to consider its internal structure for improvement, control and review of performance of respected chain. Then the usage of network DEA models will be very effect. Therefore, this paper states the evaluation of supply chain' performance regard to all members of chain under non-radial model and using procedure of SBM model with three central, decentral and mix mechanisms. Finally through example, it is shown that the usage of this model leads to the evaluation very exactly.
\end{abstract}

Keywords: Data Envelopment Analysis, Network DEA, Supply Chain Management, Performance Evaluation.

\section{Introduction}

Data Envelopment Analysis (DEA) was proposed by Charnes et al. [3], is a non-parametric linear programming technique for measuring the relative efficiency of a set Decision Making Units (DMUs), with the common set of inputs and outputs. The performance analysis is the basic element of programming, control and effective decision and can take necessary information from system for showing developments, recognition, and identification of problems and introduced to determinants of organization. Always managers have considered to DEA as suitable instrument for the evaluation of organizations' performance. This method as nonparametric method that has not need to the function of identified production, can enable the managers and directors of organizations that have corrective evaluation of self-set until have capacity of self-power and weakness points' recognition in corrective path to attain the goals. Such as the application of this method is the evaluation of supply chain's performance as productive process. The supply chain is the one of the distributive centers that its deputy is to become raw materials to intermediated produce and then final productions and its delivery among customers. Liang et al. [8] identified the efficiency of supply chain and its members through one DEA model. Chen and Liang [2] analyzed the efficiency of supply chain via one open DEA model. Easton et al. [4] introduced one DEA model for the comparison of buy section of companies in petrochemical industry. In regard to new economic-political status and markets globing and increase of rivals in global class, managers

* Corresponding author:

Somayeh Mamizadeh-Chatghayeh (Somayeh_Mamizadeh@yahoo.com) 
have to improve the self-internal process of organization for survival in rival global scene. Industrial managers have understand that material and received services of various producers have very effect on increasing capacities of organization when force to customers' needs. But in regard to supply chain which has complex structure, must be its scale of performance so that consider to its internal structure. Among various methods of evaluation, it is very useful to consider DEA methods. Sexton and Lewis [7] developed a network DEA framework which identifies the deficiency of network through deficiency of subunits. Fare and Grosskopt [5] developed a network DEA method for modeling multistep process and also Gollany et al. [6] introduced model for measure of efficiency of multiple series system. Yan and Chen [1] studied the evaluation of supply chain's performance field. The network DEA models was introduced under three different organized mechanisms; central, decentral and mix. The base of such work was a radial model under input nature.

Here, we introduced a model according to ever three mechanisms in regard to advantages of non-radial models. The implement process of this model is according to non-radial SBM model that works base of Slacks. Such as advantages of non-radial models on radial models could allude to this case. These models have identified deficiency or efficiency of a DMU through one problem solving certainly while need to two problems solving for identifying exact efficiency or deficiency in radial models. On the other hand, it needs to separate input and output nature in radial models and all inputs (outputs) decrease (increase) to one scale while it does not need such separation in non-radial models and ever input (output) decrease (increase) to self-shortage (surplus). In this path, we introduced one non-radial model for estimation of supply chain and it will be indicated to attain the most exact evaluation. To continuance, introduce non-radial model in section 2, explain the advantage of this model via examples in section 3 and finally the conclusion in section 4 respectively.

\section{Method}

We suppose n-two steps supply chains according to Figure 1. In it, $\mathrm{S}$ and $\mathrm{M}$ are provider and producer respectively. $\mathrm{X}$ is inputs vector of section $\mathrm{S}$ and $\mathrm{Y}_{1}$,
$Y_{2}$ are its outputs that are inputs vectors for $M_{1}, M_{2}$ in fact and $Z_{1}, Z_{2}$ vectors are outputs correspond to $M_{1}$, $\mathrm{M}_{2}$. Now we consider introducing of non-radial models with three mechanisms. In radial models, retraction of inputs and expansion of outputs occurred with one scale but in non-radial models do not act to this form and in ever input (output) change through self-increase (decrease) scale. For instance, non-radial models are collective model that do not compute the efficiency rate in the evaluation with this model and only identify to deficiency or efficiency of $\mathrm{DMU}_{\mathrm{S}}$ that is the weak of collective model. To be continuum, SBM model is used for development and expansion of collective models that introduce a scalar as efficiency for each unit. Then it is helped the SBM model structure and provide a model for the evaluation of supply chain's performance. In this model, surplus of inputs and shortage of outputs and also indicate the deficiency and efficiency of supply chain simultaneously.

\subsection{Non-radial Model Base on the Conception of Central Control}

In this mechanism, whole supply chain directs and controls via unique determinant. We have DEA model relates to this mechanism in regards to the introduced model as follows [1]. We evaluate $\mathrm{DMU}_{\mathrm{o}}$ under DMU and it supposes in this model that $\mathrm{Z}_{0}{ }^{1}, \mathrm{Z}_{0}{ }^{2}, \mathrm{X}_{\mathrm{o}}>0, \mathrm{~S}_{\mathrm{i}}^{-}$ $(i=1, \ldots, m)$ and $b_{k}{ }^{1+}, b_{k}{ }^{2+}$ are indicators of surplus of input and shortage of output.

$$
\min P_{\text {central }}=\frac{1-\frac{1}{m} \sum_{i=1}^{m} \frac{s_{i}^{-}}{x_{i o}}}{\left(1+\frac{1}{a} \sum_{k=1}^{a} \frac{b_{k}^{1+}}{z_{o}^{1}}\right)\left(1+\frac{1}{a} \sum_{k=1}^{a} \frac{b_{k}^{2+}}{z_{o}^{2}}\right)}
$$

s.t

$$
\begin{aligned}
& \sum_{j=1}^{n} \lambda_{j}^{1} x_{i j}+s_{i}^{-}=x_{i o} \\
& \sum_{j=1}^{n} \lambda_{j}^{1} y_{r j}^{1} \geq \sum_{j=1}^{n} \lambda_{j}^{2} y_{r j}^{1} \\
& \sum_{j=1}^{n} \lambda_{j}^{1} y_{r j}^{2} \geq \sum_{j=1}^{n} \lambda_{j}^{3} y_{r j}^{2} \\
& \sum_{j=1}^{n} \lambda_{j}^{2} z_{k j}^{1}-b_{k}^{1+}=z_{k o}^{1}
\end{aligned}
$$




$$
\begin{gathered}
\sum_{j=1}^{n} \lambda_{j}^{\mathbf{a}} z_{k j}^{2}-b_{k}^{2+}=z_{k \circ}^{2} \\
\lambda_{j}^{1}, \lambda_{j}^{2}, \lambda_{j}^{\mathbf{a}} \geq 0, j=1, \ldots, n
\end{gathered}
$$

First, fourth and fifth equations in constraints set correspond to primary input of section $\mathrm{S}$ and are to give final outputs; $\mathrm{Z}_{0}{ }^{1}, \mathrm{Z}_{\mathrm{o}}^{2}$. Second and third inequalities are corresponding to middle products in the above constraints. In this mechanism the input of section $\mathrm{M}$ should not be more than the output of section S.

Theorem 1. $\mathrm{DMU}_{\mathrm{o}}$ is the Parato-efficiency in nonradial model if and only if $\mathrm{P}^{*}=1$.

Proof Suppose $\mathrm{P}^{*}=1$, then;

$1-\frac{1}{m} \sum_{i=1}^{m} \frac{s_{i}^{*-}}{x_{i o}}=\left(1+\frac{1}{a} \sum_{k=1}^{a} \frac{b_{k}^{1 *}}{z_{o}^{1}}\right)\left(1+\frac{1}{a} \sum_{k=1}^{a} \frac{b_{k}^{2 *}}{z_{o}^{2}}\right)$

$\Rightarrow \frac{1}{a} \sum_{k=1}^{a} \frac{b_{k}^{2 *}}{z_{0}^{2}}+\frac{1}{a} \sum_{k=1}^{a} \frac{b_{k}^{1 *}}{z_{0}^{1}}+\left(\frac{1}{a} \sum_{k=1}^{a} \frac{b_{k}^{1 *}}{z_{0}^{1}}\right)\left(\frac{1}{a} \sum_{k=1}^{a} \frac{b_{k}^{2 *}}{z_{0}^{2}}\right)$

$$
+\frac{1}{m} \sum_{i=1}^{m} \frac{s_{i}^{*-}}{x_{i 0}}=0
$$

$s_{i}^{*-}=b_{k}^{1 *}=b_{k}^{2 *}=0$

Its inverse is true.

\subsection{Non-radial Model Under the Conception of Decentral Control}

In this mechanism, ever section of supply chain direct and control under unique determinant. Then we expect different strategy for ever section. Notification and precision to middle productions are obvious. Similarity, the DEA model relate to this mechanism can be stated as follows:

$$
\min P_{\text {decentral }}=\frac{1-\frac{1}{m} \sum_{i=1}^{m} \frac{s_{i}^{-}}{x_{i o}}}{\left(1+\frac{1}{a} \sum_{k=1}^{a} \frac{b_{k}^{1+}}{z_{o}^{1}}\right)\left(1+\frac{1}{a} \sum_{k=1}^{a} \frac{b_{k}^{2+}}{z_{o}^{2}}\right)}
$$

s.t

$$
\begin{gathered}
\sum_{j=1}^{n} \lambda_{j}^{1} x_{i j}+s_{i}^{-}=x_{i o} \\
\sum_{j=1}^{n} \lambda_{j}^{1} y_{r j}^{1} \geq \sum_{j=1}^{n} \lambda_{j}^{2} y_{r j}^{1}
\end{gathered}
$$

$$
\begin{gathered}
\sum_{j=1}^{n} \lambda_{j}^{1} y_{r j}^{2} \geq \sum_{j=1}^{n} \lambda_{j}^{3} y_{r j}^{2} \\
\sum_{j=1}^{n} \lambda_{j}^{2} z_{k j}^{1}-b_{k}^{1+}=z_{k \circ}^{1} \\
\sum_{j=1}^{n} \lambda_{j}^{3} z_{k j}^{2}-b_{k}^{2+}=z_{k \circ}^{2} \\
\sum_{j=1}^{n} \lambda_{j}^{2} y_{r j}^{1} \leq y_{r o}^{1} \\
\sum_{j=1}^{n} \lambda_{j}^{3} y_{r j}^{1} \leq y_{r o}^{2} \\
\lambda_{j}^{1}, \lambda_{j}^{2}, \lambda_{j}^{3} \geq 0, j=1, \ldots, n
\end{gathered}
$$

First, fourth and fifth equations in above constraints set correspond to primary input for giving final output. Second, third, sixth and seventh inequalities are corresponding to middle productions. Notification to this point has important that modification of $\mathrm{y}^{1}, \mathrm{y}^{2}$ should not more than $\mathrm{y}_{0}{ }^{1}, \mathrm{y}_{0}{ }^{2}$ for section $\mathrm{M}_{1}, \mathrm{M}_{2}$.

\section{Theorem 2.}

We indicate that

$$
P_{\text {decentral }}^{*} \leq \theta_{\text {decentral }}^{*}
$$

Proof suppose $\left(\lambda^{1 *}, \lambda^{2 *}, \lambda^{3 *}, s^{-*}, b^{1 *}, b^{2 *}, \Theta^{*}\right)$ is the optimal solution for decentral control model [1]. Replacing this optimal solution in constraints of this model;

$$
\left\{\begin{array}{c}
\sum_{j=1}^{n} \lambda_{j}^{1 *} x_{i j}+s_{i}^{*-}=\theta_{\text {decentral }}^{*} x_{i o} \\
\sum_{j=1}^{n} \lambda_{j}^{1 *} y_{r j}^{1} \geq \sum_{j=1}^{n} \lambda_{j}^{2 *} y_{r j}^{1} \\
\sum_{j=1}^{n} \lambda_{j}^{1 *} y_{r j}^{2} \geq \sum_{j=1}^{n} \lambda_{j}^{2 *} y_{r j}^{2} \\
\sum_{j=1}^{n} \lambda_{j}^{2 *} z_{k j}^{1}-b_{k}^{1 *}=z_{k o}^{1} \\
\sum_{j=1}^{n} \lambda_{j}^{2 *} z_{k j}^{2}-b_{k}^{2 *}=z_{k o}^{2}
\end{array}\right.
$$




$$
\begin{aligned}
& \sum_{j=1}^{n} \lambda_{j}^{2 *} y_{r j}^{1} \leq y_{r o}^{1} \\
& \sum_{j=1}^{n} \lambda_{j}^{2 *} y_{r j}^{1} \leq y_{r o}^{2}
\end{aligned}
$$

It can be written;

$$
\begin{gathered}
x_{i o}=\sum_{j=1}^{n} \lambda_{j}^{1 *} x_{i j}+s_{i}^{-*}+\left(1-\theta^{*}\right) x_{i o} \\
z_{k 0}^{1}=\sum_{j=1}^{n} \lambda_{j}^{2 *} z_{k j}^{1}-b_{k}^{1 *} \\
z_{k o}^{2}=\sum_{j=1}^{n} \lambda_{j}^{2 *} z_{k j}^{2}-b_{k}^{2 *}
\end{gathered}
$$

And we replace;

$$
\begin{aligned}
& \overline{\lambda^{1}}=\lambda^{1 *}, \overline{\lambda^{2}}=\lambda^{2 *}, \overline{\lambda^{3}}=\lambda^{3^{*}} \\
& \widehat{s^{-}}=s^{-\bullet}+(1-\theta) x_{i \circ} \\
& \widehat{b^{1}}=b^{1 *}, \widehat{b^{2}}=b^{2 *} .
\end{aligned}
$$

And $\quad\left(\left(\lambda^{\dagger} 1\right)^{-},\left(\lambda^{\dagger} 2\right)^{-},\left(\lambda^{\dagger} 3\right)^{-}, \quad\left(s^{\uparrow}-\right)^{\wedge},\left(b^{\uparrow} 1\right)^{\wedge}\right.$, $\left(b^{\uparrow} \mathbf{1}\right)$, is introduced a feasible solution for non-radial model. Now we get the value of objective function via replacing feasible solution:

$P_{\text {decentral }}=\frac{1-\frac{1}{m}\left\{\sum_{i=1}^{m}\left[\frac{s_{i}^{-*}}{x_{i o}}+\left(1-\theta^{*}\right)\right\}\right]}{\left(1+\frac{1}{a} \sum_{k=1}^{a} \frac{b_{k}^{1 *}}{z_{o}^{1}}\right)\left(1+\frac{1}{a} \sum_{k=1}^{a} \frac{b_{k}^{2 *}}{z_{o}^{2}}\right)}$

Therefore we have:

$$
P_{\text {decentral }} \leq \theta_{\text {decentral }}^{*}
$$

And because the problem is minimal and we have through replacing feasible solution; $p \leq \theta^{*}$, then we will have:

$$
p_{\text {decentral }}^{*} \leq \theta_{\text {decentral }}^{*}
$$

Secondary result: Above theorem states that all deficiency considers in the computation of P. In this model, weak efficiency DMUs does not show and gets to powerful efficiency through solving a model.

\subsection{Non-radial Model Under the Conception of mix Control}

In this status, it supposes that some supply chain sections control via a determinant and some other sections control through different determinants. In this paper, section $\mathrm{M}_{2}$, S control under a determinant and section $\mathrm{M}_{1}$ control via different determinant. Also in this case, we have the related DEA model to below form:

$$
\min P_{\text {mix }}=\frac{1-\frac{1}{m} \sum_{i=1}^{m} \frac{s_{i}^{-}}{x_{i o}}}{\left(1+\frac{1}{a} \sum_{k=1}^{a} \frac{b_{k}^{1+}}{z_{o}^{1}}\right)\left(1+\frac{1}{a} \sum_{k=1}^{a} \frac{b_{k}^{2+}}{z_{o}^{2}}\right)}
$$

s.t

$$
\begin{gathered}
\sum_{j=1}^{n} \lambda_{j}^{1} x_{i j}+s_{i}^{-}=x_{i o} \\
\sum_{j=1}^{n} \lambda_{j}^{1} y_{r j}^{1} \geq \sum_{j=1}^{n} \lambda_{j}^{2} y_{r j}^{1} \\
\sum_{j=1}^{n} \lambda_{j}^{1} y_{r j}^{2} \geq \sum_{j=1}^{n} \lambda_{j}^{a} y_{r j}^{2} \\
\sum_{j=1}^{n} \lambda_{j}^{2} z_{k j}^{1}-b_{k}^{1+}=z_{k o}^{1} \\
\sum_{j=1}^{n} \lambda_{j}^{a} z_{k j}^{2}-b_{k}^{2+}=z_{k o}^{2} \\
\sum_{j=1}^{n} \lambda_{j}^{2} y_{r j}^{1} \leq y_{r o}^{1} \\
\lambda_{j}^{1}, \lambda_{j}^{2}, \lambda_{j}^{a} \geq 0, j=1, \ldots, n
\end{gathered}
$$

In this model, first equation in constraints set correspond to input of section $\mathrm{S}$ and fourth, fifth equations determine the final output of supply chain. Second, third and sixth inequalities in constraints set correspond to middle productions and sixth inequality indicates the modification in input of section $\mathrm{M}_{1}$.

\section{Theorem 3.}

Now we show the relation between the efficiency in these three mechanisms:

$$
p_{\text {central }}^{*} \leq p_{\text {mix }}^{*} \leq p_{\text {decentral }}^{*}
$$




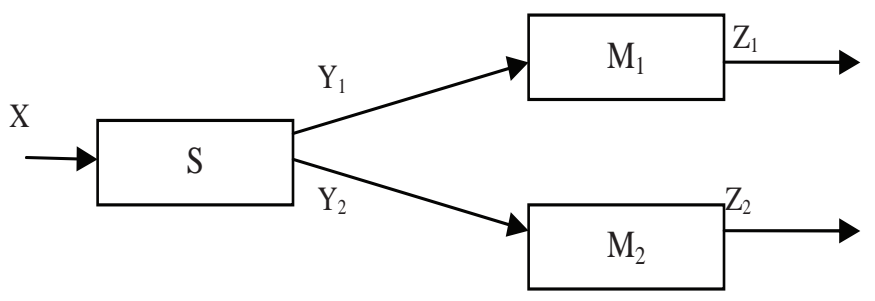

Figure 1. A supply chain.

Table 1. Input-Output data

\begin{tabular}{llllrl}
\hline$Z_{2}$ & $Z_{1}$ & $Y_{2}$ & $Y_{1}$ & \multicolumn{1}{c}{$X$} & $\mathrm{DMU}$ \\
\hline 3 & 4 & 4 & 4 & 5 & $\mathrm{DMU}_{1}$ \\
2 & 8 & 1 & 4 & 6 & $\mathrm{DMU}_{2}$ \\
6 & 2 & 3 & 1 & 5 & $\mathrm{DMU}_{3}$ \\
4 & 8 & 2 & 8 & 12 & $\mathrm{DMU}_{4}$ \\
4 & 8 & 2 & 4 & 6 & $\mathrm{DMU}_{5}$ \\
6 & 2 & 3 & 2 & 6 & $\mathrm{DMU}_{6}$ \\
2 & 8 & 1 & 8 & 6 & $\mathrm{DMU}_{7}$ \\
9 & 2 & 3 & 1 & 20.3 & $\mathrm{DMU}_{8}$ \\
3 & 17 & 1 & 8 & 6 & $\mathrm{DMU}_{9}$ \\
\hline
\end{tabular}

First we indicate:

$$
p_{\text {mix }}^{*} \leq p_{\text {decentral }}^{*}
$$

Suppose $\left(p_{1}(\text { decentral })^{\dagger} *, \lambda^{\dagger}(1 *), \lambda^{\dagger}(2 *), \lambda^{\dagger}(3 *)\right)$ is one optimal solution for decentral control model. This solution is feasible clearly for mix control model $\left(S_{\operatorname{mix}} \subset S_{\text {decentral }}\right.$ ) and similarity it can show that ever optimal solution of mix model is one feasible solution for central model.

\section{Example}

Consider a two-steps supply chains according to Figure 1. We indicate the supply chain's data in Table 1 similar

Table 2.

\begin{tabular}{ccccccc}
\hline $\mathbf{e}_{\text {decentral }}^{*}$ & $\mathbf{e}_{\text {mix }}^{*}$ & $\mathbf{e}_{\text {central }}^{*}$ & $p_{\text {decentral }}^{*}$ & $p_{\text {mix }}^{*}$ & $p_{\text {central }}^{*}$ & $\mathrm{DMU}$ \\
\hline 0.37 & 0.37 & 0.37 & 0.17 & 0.17 & 0.17 & $\mathrm{DMU}_{1}$ \\
0.49 & 0.49 & 0.49 & 0.48 & 0.48 & 0.19 & $\mathrm{DMU}_{2}$ \\
0.50 & 0.50 & 0.50 & 0.47 & 0.47 & 0.10 & $\mathrm{DMU}_{3}$ \\
0.28 & 0.28 & 0.28 & 0.20 & 0.10 & 0.06 & $\mathrm{DMU}_{4}$ \\
0.55 & 0.55 & 0.55 & 0.52 & 0.52 & 0.32 & $\mathrm{DMU}_{5}$ \\
0.41 & 0.41 & 0.41 & 0.20 & 0.20 & 0.07 & $\mathrm{DMU}_{6}$ \\
0.49 & 0.49 & 0.49 & 0.37 & 0.19 & 0.19 & $\mathrm{DMU}_{7}$ \\
0.57 & 0.57 & 0.57 & 0.17 & 0.17 & 0.01 & $\mathrm{DMU}_{8}$ \\
1.00 & 1.00 & 1.00 & 1.00 & 1.00 & 1.00 & $\mathrm{DMU}_{9}$ \\
\hline
\end{tabular}

to [1] that include of $\mathrm{DMU}_{9}$. We have shown the results of solving models $p_{\text {mix }}, p_{\text {decentral }}, p_{\text {central }}$ for the computation of supply chain's efficiency in Table 2 and also the efficiency of supply chain is cited to follow it using pervious radial model until reader be able to observe the difference of these two models easily. From the Tables it is easily understood that DMUs satisfies the pervious theorems in this paper. In the comparison of second and fifth columns in Table 2, it clears that can more exact evaluate for the evaluation of supply chain via introduced model in this paper. $\mathrm{DMU}_{9}$ shows that if a unit is the Parato-efficient form under radial model, then will have efficient in this non-radial model and also in the comparison of second, third and fourth columns indicate the accuracy of theorem 3.

\section{Conclusion}

The manager must have exact and sufficient information of organization's performance. That is, the performance of organization must be evaluated and studied exactly until manager is able to select optimal strategy for the future of self-organization. Organizations which intend to attain the cited performance, have gained the potential of supply chain's performance. The membership in supply chain along good performance will create manifest performance. Therefore, we have introduced one non-radial model via DEA in this paper until we could introduce exact information to management of supply chain in central, decentral and mix cases.

\section{References}

1. Chen C, and Yan H (2011). Network DEA model for supply chain performance evaluation, European Journal Operation Research, 213(1), 147-155.

2. Chen Y, and Liang L (2006). A DEA game model approach to supply chain efficiency, Annals of Operation Research, vol 145(1), 5-13.

3. Charnes A, Cooper W W et al. (1978). Measuring the efficiency of decision-making, European Journal of Operational Research, vol 2(6), 429-444.

4. Easton L, Murphy D J et al. (2002). Purchasing performance evaluation: with data envelopment analysis, European Journal of Purchasing \& Supply Management, vol 8(3), 123-134. 
5. Fare R, and Grosskopf S (2000). Network DEA, SocioEconomic planning Sciences, vol 34(1), 35-49.

6. Golany B, Hackman S T et al. (2003). An efficiency measurement framework for multi-stage production systems. Annals of Operation Research, vol 145(1), 51-68.
7. Lewis H F, and Sexton T R (2004). Network DEA: efficiency analysis of organizations with complex internal structure. Computers and Operation Research, vol 31(9), 1365-1410.

8. Liang L, Yang F et al. (2006). DEA models for supply chain efficiency evaluation. Annals of Operation Research, vol 145(1), 35-49. 